\title{
Identificação laboratorial de micobactérias em amostras respiratórias de pacientes HIV-positivos com suspeita de tuberculose
}

\author{
Laboratory identification of mycobacteria in respiratory samples from \\ HIV-positive patients suspected of tuberculosis
}

\author{
Liliana Aparecida Zamarioli ${ }^{1}$, Andréa Gobetti Vieira Coelho ${ }^{1}$, Clemira Martins Pereira ${ }^{1}$, \\ Lucilaine Ferrazoli ${ }^{2,3}$ e Ricardo Helbert Bammann ${ }^{3,4}$
}

\begin{abstract}
RESUMO
Foram analisados retrospectivamente os registros (2000 a 2004) do Laboratório de Microbiologia do Instituto Adolfo Lutz de Santos, SP referentes a pacientes infectados pelo virus da imunodeficiência humana com suspeita de tuberculose pulmonar. Foram encaminhadas 1.321 amostras com finalidade de diagnóstico, correspondendo a 880 casos suspeitos de tuberculose em 693 pacientes. Cento e trinta e quatro baciloscopias foram positivas e em 188 culturas houve crescimento de micobactérias, correspondendo a 161 casos confirmados. Houve identificação de Mycobacterium tuberculosis em 126 (78,3\%) e micobactérias não tuberculosas em 39 (24,2\%). Em quatro casos, houve concomitância de Mycobacterium tuberculosis e micobactérias não tuberculosas (porém em amostras distintas). 0 perfil de sensibilidade às drogas antituberculose revelou 18 (14,3\%) casos de resistência a pelo menos um medicamento. Estes resultados reforçam a necessidade de submeter à rotina laboratorial completa - baciloscopia, cultura com identificação e testes de sensibilidade às drogas - as amostras respiratórias de pacientes soropositivos para o vírus da imunodeficiência humana com suspeita de tuberculose para direcionamento terapêutico adequado.
\end{abstract}

Palavras-chaves: Tuberculose pulmonar. Tuberculose resistente a múltiplos medicamentos. Micobactérias atípicas. Síndrome de imunodeficiência adquirida.

\section{ABSTRACT}

The records (2000 to 2004) of the Microbiology Laboratory of the Adolfo Lutz Institute in Santos, Brazil, were retrospectively analyzed regarding patients infected with the human immunodeficiency virus (HIV) and suspected of pulmonary tuberculosis. 1,321 samples for diagnosis purposes were selected, corresponding to 880 suspected tuberculosis cases in 693 patients. There were 134 smear-positive samples and mycobacteria growth occurred in 188 cultures, corresponding to 161 confirmed cases. Mycobacterium tuberculosis was identified in 126 (78.3\%) and non-tuberculous mycobacteria in 39 (24.2\%). In four cases, both Mycobacterium tuberculosis and non-tuberculous mycobacteria were simultaneously recovered from different samples. The profile of sensitivity to anti-tuberculosis drugs revealed 18 (14.3\%) cases of resistance to at least one drug. These results reinforce the need to carrying out the complete laboratorial routine (sputum smear microscopy, culture and susceptibility to antituberculous drugs) for respiratory samples from human immunodeficiency virus-positive patients with suspected tuberculosis in order to direct appropriate therapy

Key-words: Pulmonary tuberculosis. Multidrug-resistant tuberculosis. Atypical mycobacteria. Acquired immunodeficiency syndrome.

Tuberculose (TB) e infecção pelo virus da imunodeficiência humana (HIV) formam uma combinação de alta letalidade. A tuberculose é a principal causa de morte entre os indivíduos HIVpositivos, concorrendo com $13 \%$ das mortes por aids no mundo ${ }^{42}$.

\footnotetext{
1. Laboratório de Microbiologia, Laboratório I de Santos, Instituto Adolfo Lutz, Santos, SP. 2. Laboratório de Micobactérias, Instituto Adolfo Lutz, São Paulo, SP. 3. Programa de Pós-Graduação da Coordenadoria de Controle de Doenças da Secretaria Estadual da Saúde, São Paulo, SP. 4. Instituto de Infectologia Emílio Ribas, São Paulo, SP. Endereço para correspondência: Dra. Liliana A. Zamarioli. Rua Silva Jardim 90, Vila Nova, 11015-020 Santos, SP.

Telefax: $55133232-5112$

e-mail: lilizy@globo.com

Recebido para publicação em 05/08/2008

Aceito em 29/04/2009
}

Um estudo epidemiológico da coinfecção TB/HIV conduzido por Kritski e cols ${ }^{22}$ no Rio de Janeiro entre 1987 e 1992 revelou que o índice de coinfecção foi de aproximadamente 5,6\% do total de casos de TB. Dentre o total de casos notificados em São Paulo, entre 1998 e 2006, 16\% eram HIV-positivos ${ }^{30}$.

A Baixada Santista apresenta uma das maiores prevalências de aids no país, ocupando a segunda posição no Estado de São Paulo, o que propicia a ocorrência de altas taxas de coinfecção TB/HIV (12\% do total de casos de TB no ano de 2004) ${ }^{32}$. Apresentando esse quadro epidemiológico a região é considerada prioritária para o Programa de Controle da Tuberculose (PCT) da Secretaria de Estado da Saúde no que se refere às medidas e ações de vigilância em saúde pública. Para desenvolver as atividades do PCT, o município de Santos conta com um laboratório regional 
de referência no diagnóstico da TB (o Instituto Adolfo Lutz - IAL) e uma unidade assistencial organizada e especializada (o Centro de Referência em AIDS de Santos - CRAIDS).

Foram objetivos deste estudo avaliar o diagnóstico laboratorial obtido por estes serviços diante da suspeita de TB pulmonar em pacientes infectados pelo HIV, comparando os resultados da baciloscopia com os resultados da cultura de amostras de escarro, quantificando a identificação de micobactérias não tuberculosas (MNT) e determinando o perfil de resistência das cepas de Mycobacterium tuberculosis (Mtb) aos quimioterápicos utilizados no tratamento específico.

\section{MATERIAL E MÉTODOS}

Trata-se de uma análise retrospectiva dos dados da rotina laboratorial de amostras respiratórias encaminhadas ao IALSantos, setor de micobactérias, no período de janeiro de 2000 a dezembro de 2004.

As fontes de informações deste estudo foram as requisições médicas para coleta de baciloscopia do escarro, o Livro de Registro de Baciloscopia e Cultura de TB do IAL-Santos e a respectiva ficha de acompanhamento do paciente contendo dados clínicos de relevância, aberta no laboratório para todos cujas amostras foram enviadas para diagnóstico, independente do resultado bacteriológico final.

As amostras incluídas neste estudo foram somente do tipo escarro, todas de pacientes HIV positivos com 15 anos de idade ou mais, de ambos os sexos, matriculados e atendidos no CRAIDS. Todos os pacientes tinham sorologia positiva para HIV com diagnóstico confirmado pela técnica de Western-Blot.

As técnicas laboratoriais utilizadas foram as rotineiramente utilizadas pelo Serviço, padronizadas conforme normas e recomendações descritas no Manual de Bacteriologia da $\mathrm{TB}^{16}$.

Baciloscopia. Coloração dos esfregaços pelo método de Ziehl-Neelsen após tratamento pela descontaminação de Petroff.

Cultura. Semeadura em meio de Löwenstein-Jensen após digestão e descontaminação de Petroff, incubação a $37^{\circ} \mathrm{C}$ por até 60 dias, com inspeção semanal.

Identificação e teste de sensibilidade às drogas. Segundo a rotina do laboratório, culturas positivas são encaminhadas ao IAL São Paulo. As micobactérias são então identificadas como Mtb e MNT por meio de suas propriedades bioquímicas e fenotípicas ${ }^{16}$. Os testes de avaliação de sensibilidade às drogas antiTB são realizados pelo método da razão de resistência, utilizando meios com concentrações padronizadas de antibióticos específicos. Para este estudo considerou-se apenas o perfil de sensibilidade a três destas drogas (isoniazida, rifampicina e pirazinamida) ${ }^{16}$.

Amostras que não seguiram toda a metodologia proposta e aquelas contaminadas e/ou com crescimento de raras colônias de microrganismos não identificáveis foram excluidas da casuística.
Neste trabalho foram adotadas as seguintes definições:

Caso suspeito. Para definir quantas destas amostras realmente corresponderam a um episódio individual de suspeita clínica de TB, foram discriminados os homônimos através do agrupamento dos pacientes em ordem alfabética e sua respectiva data de nascimento, além das datas de registro no laboratório. Considerou-se que uma amostra de um mesmo paciente encaminhada ao laboratório após intervalo maior do que seis meses em relação a outra amostra cuja cultura havia sido negativa representava, na verdade, um novo episódio clínico, um novo caso suspeito de TB. Da mesma forma considerou-se que uma amostra de um mesmo paciente encaminhada ao laboratório (com finalidade diagnóstica) após intervalo maior do que doze meses em relação a outra amostra que havia definido o diagnóstico de micobacteriose (através da cultura positiva) significava também um outro episódio clínico, um outro caso suspeito de TB.

Cepa. Todo isolado bacteriano identificado em cultura nas amostras de um mesmo caso suspeito. Caso várias culturas de um mesmo caso suspeito tenham sido positivas, considerouse como sendo a mesma cepa todas as micobactérias que apresentassem resultado idêntico no teste de identificação ou teste de sensibilidade às drogas.

0 cálculo das taxas de sensibilidade e especificidade da baciloscopia consideraram como padrão ouro o resultado da respectiva cultura de micobactérias.

Com base na Resolução nº 196 de 10/10/1996 do Conselho Nacional de Saúde para Pesquisa Científica em Seres Humanos, os dados publicados não permitem identificar as pessoas cujas amostras foram incluídas na pesquisa. Este estudo teve a aprovação do Comitê de Ética em Pesquisa do Instituto Adolfo Lutz, registrado sob o número 028/2005. Não houve patrocínio ou ajuda financeira institucional em nenhum momento da pesquisa.

\section{RESULTADOS}

Inicialmente, foram incluídas 1.510 amostras de escarro de pacientes HIV-positivos examinadas no IAL-Santos durante o período de 2000 a 2004. Destas, 35 (2,3\%) foram excluídas devido a contaminação ou crescimento de raras colônias de microrganismos não identificáveis. Das amostras remanescentes, $154(10,2 \%)$ foram excluídas por terem sido encaminhadas ao laboratório para controle de tratamento. Restaram, portanto, $1.321(87,5 \%)$ amostras encaminhadas ao laboratório para fins diagnósticos de TB pulmonar.

Entre as 1.321 amostras, foram identificados 880 casos suspeitos de TB pulmonar tal como definido na metodologia do estudo, correspondendo a 693 pacientes, todos HIV-positivos $431(62,2 \%)$ eram homens, sendo a faixa etária entre 30 e 49 anos de idade a mais $(69,7 \%)$ prevalente.

0 número de amostras encaminhadas ao laboratório por cada caso suspeito variou entre um e oito, com mediana de 1,3. Dos 880 casos suspeitos, 579 (65,8\%) foram representados por uma única amostra de escarro encaminhada ao laboratório. 
Do total de 1.321 baciloscopias realizadas com finalidade diagnóstica, o resultado foi negativo em 1.187 (89,9\%) e positivo em $134(10,1 \%)$ amostras. Do total de 134 baciloscopias positivas, $117(87,4 \%)$ correspondiam à primeira amostra de determinado paciente, $16(11,9 \%)$ eram a segunda amostra e uma $(0,7 \%)$ terceira amostra também foi positiva.

Com referência aos 880 casos suspeitos de TB pulmonar, $768(87,3 \%)$ apresentaram todas as baciloscopias negativas e $112(12,7 \%)$ apresentaram pelo menos uma baciloscopia positiva. Destes 112, a baciloscopia foi positiva já na primeira amostra em 111 (99,1\%) casos. Dentre os 301 casos suspeitos, com mais de uma amostra encaminhada ao laboratório, a segunda coleta de escarro acrescentou somente um (0,3\%) diagnóstico. Três ou mais amostras não representaram acréscimo algum à positividade obtida pelas duas coletas que as antecederam.

Das 1.321 amostras de escarro, 1.133 (85,8\%) culturas foram negativas e $188(14,2 \%)$ foram positivas. Na Figura 1, encontra-se a distribuição da quantificação da carga bacilar na pesquisa direta em função das culturas positivas, conforme 0 índice baciloscópico recomendado pelo Ministério da Saúde ${ }^{15}$. Das 188 amostras com cultura positiva, 185 (98,4\%) foram positivas já na primeira amostra; houve um acréscimo de três $(1,6 \%)$ culturas positivas ao se considerar os resultados da segunda amostra de escarro. Não houve acréscimo na positividade com a terceira amostra.

A distribuição dos resultados obtidos pela baciloscopia e/ou pela cultura em relação às 1.321 amostras e aos 880 casos suspeitos está demonstrada na Tabela 1.

A análise estatística dos resultados obtidos na Tabela $\mathbf{1}$ permitiu calcular os índices de sensibilidade $(60,1 \%)$ e de especificidade $(98,1 \%)$ para a baciloscopia quando comparada com a cultura (padrão ouro).

Dentre os 161 casos suspeitos de TB em que houve crescimento de micobactérias na cultura, 126 (78,3\%) foram identificadas como Mtb enquanto em 39 (24,2\%) foram identificadas MNT (em quatro amostras houve crescimento simultâneo de duas espécies de MNT). Em outros quatro casos, houve concomitância de crescimento de Mtb e MNT (em amostras distintas, porém contemporâneas). No total, foram identificadas 169 cepas de micobactérias. A identificação das espécies e a distribuição destes resultados encontra-se na Tabela 2.

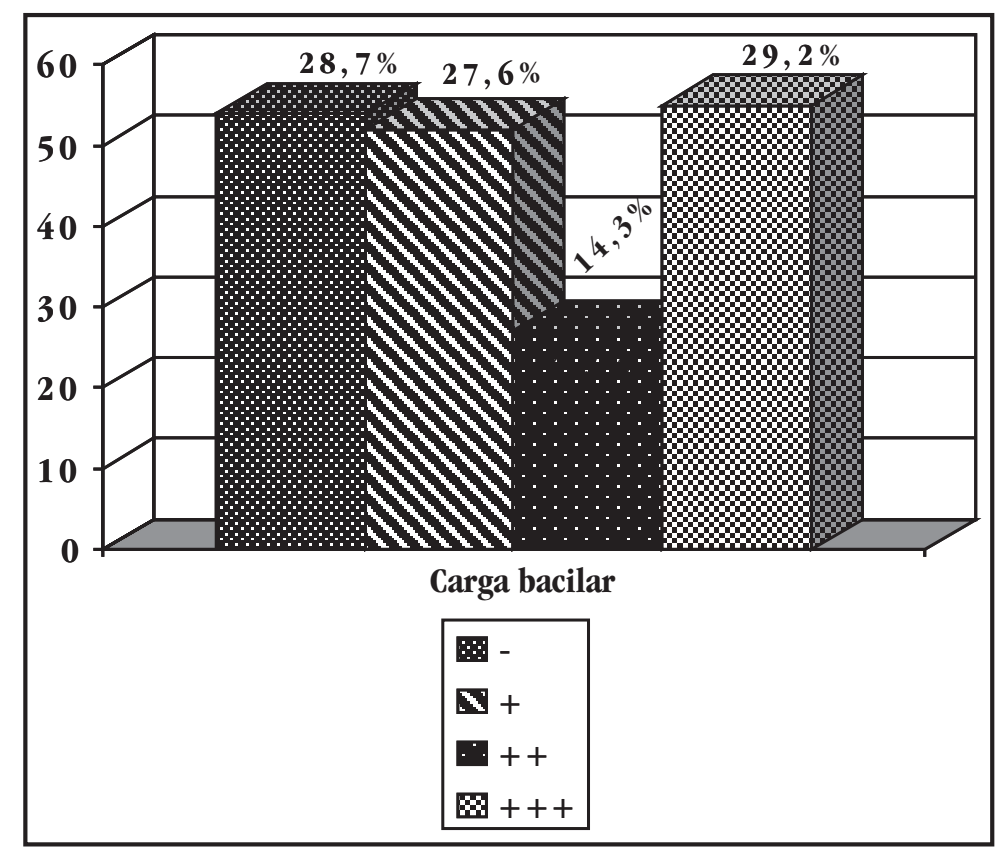

FIGURA 1

Distribuição da carga bacilar nas 188 amostras cujas culturas foram positivas (IAL- Santos, 2000-2004).

TABELA 1

Número de amostras e de casos suspeitos de tuberculose pulmonar com respectivos resultados da baciloscopia e cultura em pacientes HIV-positivos (IAL-Santos, 20002004).

\begin{tabular}{|c|c|c|c|c|}
\hline \multirow[b]{2}{*}{ Método laboratorial } & \multicolumn{2}{|c|}{ Amostras } & \multicolumn{2}{|c|}{$\begin{array}{c}\text { Casos } \\
\text { suspeitos }\end{array}$} \\
\hline & $\mathrm{n}^{0}$ & $\%$ & $\mathrm{n}^{0}$ & $\%$ \\
\hline Baciloscopia e cultura positivas & 113 & 8,5 & 94 & 10,7 \\
\hline Baciloscopia negativa e cultura positiva & 75 & 5,7 & 67 & 7,6 \\
\hline Baciloscopia positiva e cultura negativa & 21 & 1,6 & 18 & 2,0 \\
\hline Baciloscopia e cultura negativas & 1112 & 84,2 & 701 & 79,7 \\
\hline Total & 1,321 & 100,0 & 880 & 100,0 \\
\hline
\end{tabular}


Dentre os 39 casos suspeitos de TB em que houve crescimento de MNT, somente um caso pôde ser confirmado pelos critérios da American Thoracic Society (ATS) ${ }^{1}$. Observou-se que em $36(92,3 \%)$ casos as MNT foram isoladas em amostra única, em três $(7,7 \%)$ ocasiões estas foram repetidamente isoladas em duas amostras (apenas um com baciloscopia positiva) e não se observou o isolamento de MNT em três amostras consecutivas neste estudo.
Com relação aos 126 casos em que foram isoladas Mtb em cultura, 108 (85,7\%) cepas eram sensíveis às drogas testadas. As demais $18(14,3 \%)$ cepas apresentavam resistência a pelo menos um dos medicamentos. Em três $(2,4 \%)$ casos, havia resistência a três drogas, caracterizando a TB multirresistente (TB-MDR). A Tabela 3 revela o perfil de resistência às drogas anti-TB. A Tabela 4 apresenta dados laboratoriais referentes à identificação de Mycobacterium tuberculosis e MNT de diversos estudos científicos brasileiros.

TABELA 2

Frequiência das espécies de micobactérias isoladas em cultura de escarro de pacientes HIV-positivos distribuídas pelo número de amostras e pelo número de casos suspeitos de tuberculose pulmonar (IAL-Santos, 2000-2004).

\begin{tabular}{|c|c|c|c|c|c|c|}
\hline \multirow[b]{3}{*}{ Espécie } & \multicolumn{6}{|c|}{ Casos } \\
\hline & \multicolumn{2}{|c|}{ Amostras } & \multicolumn{2}{|c|}{ suspeitos } & \multicolumn{2}{|c|}{ Cepas } \\
\hline & $n^{\circ}$ & $\%$ & $\mathrm{n}^{0}$ & $\%$ & $\mathrm{n}^{0}$ & $\%$ \\
\hline Complexo Mycobacterium tuberculosis & 146 & 77,7 & $126^{*}$ & 78,3 & 126 & 74,5 \\
\hline Micobactérias não tuberculosas $s p$ & 12 & 6,4 & 11 & 6,8 & 11 & 6,5 \\
\hline Mycobacterium avium & 6 & 3,2 & 6 & 3,7 & 6 & 3,5 \\
\hline Mycobacterium fortuitum & 6 & 3,2 & 6 & 3,7 & 6 & 3,5 \\
\hline Mycobacterium fortuitum + Mycobacterium chelonae ${ }^{* * *}$ & 2 & 1,1 & 2 & 1,2 & 4 & 2,4 \\
\hline Mycobacterium kansasii & 5 & 2,7 & 4 & 2,5 & 4 & 2,4 \\
\hline CLA & 3 & 1,6 & 3 & 1,9 & 3 & 1,8 \\
\hline CLE & 2 & 1,1 & 2 & 1,2 & 2 & 1,2 \\
\hline Mycobacterium shimode $i$ & 2 & 1,1 & 1 & 0,6 & 1 & 0,6 \\
\hline Mycobacterium triviale + Mycobacterium terrae $e^{* * *}$ & 2 & 1,1 & 2 & 1,2 & 4 & 2,4 \\
\hline CRE & 1 & 0,5 & 1 & 0,6 & 1 & 0,6 \\
\hline Mycobacterium peregrinum & 1 & 0,5 & 1 & 0,6 & 1 & 0,6 \\
\hline Total & 188 & 100,0 & 161 & 100,0 & 169 & 100,0 \\
\hline \multicolumn{7}{|c|}{ *em quatro casos suspeitos houve concomitância de crescimento de MTb e MNT } \\
\hline \multicolumn{7}{|c|}{ **crescimento simultâneo de duas espécies de micobactérias não tuberculosas } \\
\hline \multicolumn{7}{|l|}{ CLA: micobactérias de crescimento lento acromógenas } \\
\hline \multicolumn{7}{|l|}{ CLE: micobactérias de crescimento lento escotocrormógenas } \\
\hline CRE: micobactérias de crescimento rápido escotocrormóger & & & & & & \\
\hline
\end{tabular}

TABELA 3

Perfil de resistência às drogas testadas nos casos de tuberculose confirmada por cultura em pacientes HIV-positivos (IALSantos, 2000 - 2004)

\begin{tabular}{lcc}
\hline Teste de sensibilidade & Número & Percentagem \\
\hline Mycobacterium tuberculosis resistente & 18 & 14,3 \\
Isoniazida + rifampicina + pirazinamida & 3 & 2,4 \\
Isoniazida + rifampicina & 4 & 3,2 \\
Isoniazida & 9 & 7,1 \\
Rifampicina & 2 & 1,6 \\
Pirazinamida & 0 & 0 \\
Mycobacterium tuberculosis sensível & 108 & 85,7 \\
\hline Total & $\mathbf{1 2 6}$ & $\mathbf{1 0 0 , 0}$ \\
\hline
\end{tabular}


TABELA 4

Resumo de trabalhos científicos brasileiros realizados exclusivamente com pacientes HIV-positivos apresentando os respectivos percentuais encontrados de infecção por micobactérias não tuberculosas e por Mycobacterium tuberculosis resistente a pelo menos uma droga antituberculose.

\begin{tabular}{|c|c|c|c|c|c|}
\hline Autores & $\begin{array}{c}\text { Ano da coleta de } \\
\text { dados }\end{array}$ & Local do estudo & $\begin{array}{l}\text { Número } \\
\text { de casos }\end{array}$ & $\begin{array}{l}\text { MNT } \\
(\%)\end{array}$ & $\begin{array}{c}\text { Resistência do Mtb a pelo menos } \\
\text { uma droga (\%) }\end{array}$ \\
\hline \multicolumn{5}{|c|}{ São José do Rio Preto/SP } & 17,5 \\
\hline Marques e $\operatorname{cols}^{24}$ & 2000-2006 & $\begin{array}{c}\text { LabRef, } \\
\text { Mato Grosso Sul }\end{array}$ & 67 & $\mathrm{NA}$ & 25,4 \\
\hline Zamarioli e cols* & 2000-2004 & $\begin{array}{c}\text { LabRef, } \\
\text { Santos/SP }\end{array}$ & 161 & 24,2 & 14,3 \\
\hline Bammann e cols ${ }^{2}$ & $2000-2001$ & $\begin{array}{c}\text { HospRef, } \\
\text { São Paulo/SP }\end{array}$ & 348 & 20,4 & 11,9 \\
\hline Santi e cols ${ }^{31}$ & $1999-2001$ & $\begin{array}{c}\text { HospGeral, } \\
\text { Belo Horizonte/MG }\end{array}$ & 39 & 15,4 & 21,2 \\
\hline Wolfart e cols ${ }^{40}$ & $1997-2003$ & $\begin{array}{c}\text { HospGeral, } \\
\text { Porto Alegre/RS }\end{array}$ & 398 & $\mathrm{NA}$ & 17,8 \\
\hline Coelho e cols ${ }^{9}$ & $1997-2000$ & $\begin{array}{l}\text { LabRef, } \\
\text { Santos/SP }\end{array}$ & 216 & 11,6 & NA \\
\hline Boffo e cols & $1997-2000$ & $\begin{array}{c}\text { HospRef } \\
\text { Rio Grande/RS }\end{array}$ & 28 & $\mathrm{NA}$ & 0 \\
\hline Brito e cols 8 & 1996-1998 & $\begin{array}{c}\text { HospRef, } \\
\text { Rio de Janeiro/RJ }\end{array}$ & 52 & $\mathrm{NA}$ & 28,8 \\
\hline Gomes e cols ${ }^{18}$ & 1995-1996 & $\begin{array}{c}\text { HospRef, } \\
\text { Florianópolis/SC }\end{array}$ & 39 & 7,7 & 13,9 \\
\hline Rozman e cols ${ }^{29}$ & 1993-2003 & $\begin{array}{c}\text { LabRef, } \\
\text { Baixada Santista/SP }\end{array}$ & 301 & $\mathrm{NA}$ & 18,9 \\
\hline Pinto e $\operatorname{cols}^{28}$ & 1992-1994 & $\begin{array}{c}\text { HospRef, } \\
\text { São Paulo/SP }\end{array}$ & 228 & $\mathrm{NA}$ & 20.6 \\
\hline
\end{tabular}

*dados referentes ao presente trabalho.

Mtb: Mycobacterium tuberculosis, MNT: micobactérias não tuberculosas, NA: não avaliado, LabRef: Laboratório de Referência em tuberculose, HospRef: Hospital de Referência em AIDS, HospGeral: Hospital Geral.

\section{DISCUSSÃo}

A baciloscopia é considerada o método de escolha para 0 diagnóstico da TB pulmonar ao demonstrar mais rapidamente a presença do agente etiológico e desta forma agilizar a implantação de ações visando eliminar os focos infecciosos da doença. Entretanto, no contexto da coinfecção TB/HIV a cultura de micobactérias é fundamental por sua maior positividade, por permitir a diferenciação das espécies de micobactérias e por viabilizar o teste de sensibilidade aos medicamentos antiTB ${ }^{1533536}$.

Apesar das recomendações oficiais ${ }^{16}$ de se coletar pelo menos duas amostras de escarro para aumentar significativamente 0 rendimento de casos positivos, 0 alto $(65,8 \%)$ percentual observado nesta casuística de casos suspeitos de TB representados por uma única amostra de escarro enviada ao laboratório é bastante insatisfatório e preocupante, potencialmente diminuindo as chances de diagnosticar a doença na população estudada. Este é, por um lado, o primeiro dos aspectos que merecem análise crítica dos resultados obtidos no presente estudo. Por outro lado, reflete nada mais do que a realidade e a rotina do sistema de saúde em nosso meio.
Ao se comparar o número de amostras enviadas por paciente e o número de casos com baciloscopia positiva, entretanto, notouse que a primeira amostra detectou a maioria $(99,1 \%)$ dos casos positivos e a segunda amostra adicionou apenas mais um ( $0,9 \%)$ caso. Não houve acréscimo no número de diagnósticos firmados pela terceira amostra. Ozkutuk e $\operatorname{cols}^{26}$, em estudo realizado na Turquia, detectaram uma positividade de $97 \%$ na primeira amostra, $3 \%$ na segunda e a terceira amostra também não apresentou valor diagnóstico adicional. Ipuge e cols ${ }^{20}$, na Tanzânia, obtiveram uma taxa de baciloscopias positivas na primeira amostra em $83,4 \%$ dos casos, na segunda em $12 \%$ e na terceira amostra em mais $4,4 \%$. Figueiredo ${ }^{13} \mathrm{em}$ sua tese, avaliando usuários das unidades básicas de saúde da Cidade de Taubaté/SP, obteve um percentual de 91,9\% na positividade da primeira amostra, acrescido de $6,8 \%$ com a segunda amostra e mais $1,3 \%$ com a terceira.

0 alto $(99,1 \%)$ índice de positividade obtido já pela primeira amostra no presente trabalho pode significar uma eventual maior gravidade destes casos no momento do diagnóstico, devendo o mesmo estar ocorrendo mais tardiamente e sendo os pacientes, portanto, mais bacilíferos. Neste sentido, corrobora também o fato de, com relação à carga ou intensidade bacilar das 188 amostras 
com cultura positiva (Figura 1), 43,5\% das baciloscopias terem sido caracterizadas como duas ou três cruzes. Trata-se de resultado conflitante com o conceito difundido pela literatura internacional de que pacientes com aids costumam ser paucibacilíferos ${ }^{17} 41$, embora no Brasil os aspectos epidemiológicos e terapêuticos indiquem um maior percentual de infecção pós-primária justificando repercussões na biodiversidade das populações bacilares e na comunicabilidade dos bacilos ${ }^{25} 37$.

Nas formas pulmonares cavitárias da TB, ricas em bacilos, a sensibilidade da baciloscopia é descrita como em torno de $80 \%{ }^{5}$. Nas formas intersticiais, a baciloscopia apresenta sensibilidade inferior a $32 \%{ }^{19}$. No contexto da coinfecção TB/HIV, a sensibilidade da baciloscopia encontrada nesta pesquisa $(60,1 \%)$ está abaixo dos valores de $77,5 \%$ e $62,1 \%$, respectivamente, descritos por Figueiredo ${ }^{13}$ e Coelho e cols ${ }^{9}$. Já a especificidade, da baciloscopia depende da prevalência das micobactérias na população, mas também da qualificação técnica do laboratório. A alta $(98,1 \%)$ especificidade da baciloscopia encontrada nesta casuística é similar à de outras publicações, onde os valores são sempre superiores a $90 \%{ }^{910}$.

Apesar da baciloscopia representar o principal recurso diagnóstico da TB, suas limitações demandam a realização da cultura, especialmente no contexto da coinfecção TB/HIV, onde as proporções são diferentes ${ }^{9}$. A positividade da baciloscopia no presente estudo, por exemplo, foi de 10,1\% nas 1.321 amostras de diagnóstico examinadas e de $12,7 \%$ entre os 880 casos suspeitos de TB. A positividade alcançada pela cultura de escarro foi de 14,2\% das amostras e 18,3\% dos casos suspeitos - um acréscimo de mais de $40 \%$.

Froes e cols ${ }^{14}$ em Minas Gerais obtiveram um acréscimo de 19,4\% no diagnóstico da TB com a realização da cultura; Boffo e cols ${ }^{6}$ alcançaram um rendimento maior da cultura em 10,2\% no diagnóstico de sintomáticos respiratórios; e Liberato e cols ${ }^{23}$, no nordeste brasileiro, verificaram acréscimo de 10,6\% na positividade da cultura em relação à baciloscopia do escarro de pacientes HIV-positivos.

A importância da cultura de micobactérias reflete-se também na identificação das espécies, principalmente nos pacientes imunossuprimidos, como os HIV-positivos. Além de estar relacionada ao aumento das taxas globais de incidência da TB, a pandemia da aids é a principal responsável pelo aumento da incidência de doenças pulmonares por MNT, principalmente as causadas pelo complexo Mycobacterium avium ${ }^{32}$. Devido à baixa virulência dessas espécies, sua patogenicidade está relacionada a uma maior deficiência imunológica do hospedeiro. Este comportamento epidemiológico pôde também ser observado na população deste estudo, onde dos 161 casos diagnosticados com isolamento de micobactérias, em 126 (78,3\%) foram isoladas cepas do complexo Mycobacterium tuberculosis e em $39(24,2 \%)$ casos houve crescimento de MNT. Em quatro ocasiões houve concomitância de Mycobacterium tuberculosis e MNT (em amostras distintas, porém contemporâneas).

As principais espécies de MNT aqui identificadas foram Mycobacterium avium, Mycobacterium fortuitum $\mathrm{e}$ Mycobacterium kansasii, dados semelhantes aos encontrados por outros autores no Brasil, nos EUA e no Japão ${ }^{1139}$.
Os critérios diagnósticos adotados pela ATS $^{1}$ para diagnóstico de infecções causadas por MNT enfatizam o isolamento da mesma espécie em múltiplas amostras. Sendo assim, seriam necessárias pelo menos três amostras de escarro (ou outros espécimes de sítios não estéreis) com duas culturas positivas e uma baciloscopia positiva coletadas num período de um ano ${ }^{1133}$.

Como já mencionado, o alto índice de casos suspeitos nesta casuística representados por uma única amostra de escarro inviabiliza, contudo, a adoção desses critérios. Na verdade, se forem seguidos à risca os critérios da ATS ${ }^{1}$ para o diagnóstico laboratorial de doença causada por MNT, apenas um caso seria confirmado como tal. Surge portanto a dúvida quanto ao diagnóstico destes casos, uma vez que um único isolamento pode significar simples colonização. A prática clínica corresponde a uma situação em que o médico se encontra frente a um paciente imunodeprimido (HIV-positivo) com suspeita clínica ou radiológica de TB. Se porventura a cultura de escarro solicitada por este profissional identificar uma MNT, mesmo que a rigor não confirmado laboratorialmente pelos critérios da ATS $^{1}$ ou qualquer outro a ser adotado, é pouco provável que este resultado seja desprezado e não implique numa conduta terapêutica específica.

0 teste de sensibilidade aos medicamentos anti-TB tem grande importância ao apontar alternativas terapêuticas eficientes e, neste sentido, o laboratório de referência mais uma vez cumpre papel relevante no fluxo de procedimentos e informações necessárias para o diagnóstico, tratamento e controle da TB, especialmente na coinfecção TB/HIV.

A resistência primária é devida à transmissão facilitada por doentes portadores de cepas já resistentes a indivíduos virgens de tratamento. No entanto, no Brasil a resistência pós-primária tem sido apontada como mais freqüente e determinante, inclusive em pacientes HIV-positivos ${ }^{25} 2837$. Ambas refletem a alta frequiência de tratamento irregular e de abandono existentes em nosso meio. Telles e cols ${ }^{38}$, ao analisarem os diversos fatores de risco para TB multirresistente (TB-MR) numa comunidade urbana do município de São Paulo, relataram forte associação com o fato de o paciente ter tido TB no passado e ter sido hospitalizado 24 meses antes do diagnóstico de TB; não houve associação entre TB-MR e a coinfecção TB-HIV, conforme apresentado também por outros estudos brasileiros ${ }^{482127}$. Neste sentido, é possível imaginar que 0 risco de uma grande epidemia de TB-MR no Brasil é baixo ${ }^{27}$. Neste estudo, são apresentados 126 casos diagnosticados de infecção por Mycobacterium tuberculosis, a maioria (85,7\%) deles sensível a todas as drogas testadas (Tabela 3). Em 18 (14,3\%) casos todavia foi demonstrada resistência a pelo menos uma droga antiTB. Em quatro (3,2\%) casos, houve resistência simultânea à isoniazida e à rifampicina. Em três $(2,4 \%)$ casos houve resistência a três drogas, caracterizando-se a TB-MR.

Visando confrontar, didaticamente, os resultados dos isolamentos de micobactérias não tuberculosas e de identificação de Mycobacterium tuberculosis resistentes às drogas obtidas por vários estudos realizados em diversas regiões do Brasil, os listamos na Tabela 4. Observa-se que os respectivos resultados obtidos não são demasiadamente díspares entre si e revelam números 
bastante preocupantes em termos diagnósticos e implicações terapêuticas. Embora sejam muitas as diferenças metodológicas encontradas nessas publicações, deixando inclusive difícil e incoveniente a comparação de estudos de base populacional com estudos laboratoriais, vale ressaltar que pelo menos um quinto dos pacientes HIV-positivos com suspeita de TB estão potencialmente sujeitos a um tratamento que pode ser considerado como ineficaz, se mantivermos como base o esquema tríplice preconizado no Brasil. Isto se deve tanto pelos percentuais aferidos de resistência a pelo menos uma destas três drogas, quanto pela proporção de MNT identificadas em cultura. São obviamente agravantes a esta situação: 0 atraso no atendimento e na suspeição diagnóstica, dificuldades na comunicação entre laboratórios e a assistência clínica, a falta de controle e supervisão sobre o resultado final da cultura e o teste de sensibilidade e, sobretudo, a prescrição de tratamento empírico sem aguardar e/ou confirmar o diagnóstico de certeza.

Concluindo, é fundamental que os formuladores de políticas públicas para a saúde percebam o papel crucial dos laboratórios, mas é igualmente indispensável incluir todos os setores envolvidos na luta contra a TB em uma harmoniosa integração multidisciplinar abrangendo a assistência clínica, laboratorial e social.

\section{AGRADECIMENTO}

Agradecemos à colega Dra. Maria Cecilia Outeiro Gorla do Instituto Adolfo Lutz, da Seção de Bacteriologia, pelo apoio e auxílio durante a execução do projeto.

\section{REFERÊNCIAS}

1. American Thoracic Society. An official ATS/IDSA statement: diagnosis, treatment, and prevention of nontuberculous mycobacterial diseases. American Journal of Respiratory Critical Care Medicine 175:367-416, 2007.

2. Bammann RH, Vázquez CMP, Haddad DJ, Costa JMS, Souza SA, Souza-Pinto V. Mycobacteria other than tuberculosis isolated in 6781 consecutive respiratory samples. International Journal of Infectious Diseases 8:\$50, 2004

3. Barreto AMW, Campos CED. Micobactérias não tuberculosas no Brasil. Boletim de Pneumologia Sanitária 8:23-32, 2000.

4. Barroso EC, Mota RMS, Morais MFM, Campelo CL, Barroso JB, Rodrigues JLN. Fatores associados aos tratamentos inadequados em grupo de portadores de tuberculose multirresistente. Jornal Brasileiro de Pneumologia 29:350-357, 2003.

5. Bethlem N, Bethlem EP, Ribeiro SN, Gerhardt Filho G, Silva JRL, Souza GRM, Côrrea JC, Magarão SL, Gontijo Filho PP, Fonseca LS, Sant'anna CC, Hijar MA. Tuberculose. $I n$ : Bethlem N (ed) Pneumologia. $4^{\mathrm{a}}$ edição. Editora Atheneu São Paulo, p 379-448,1995.

6. Boffo MMS, Mattos IG, Ribeiro MO, Jardim S, Souza VC. Diagnóstico laboratorial da tuberculose na cidade do Rio Grande do Sul, RS, Brasil. Revista Brasileira de Análises Clínicas 35:35-38, 2003.

7. Boffo MMS, Mattos IG, Ribeiro MO, Oliveira Neto IC. Tuberculosis associated to AIDS: demographic, clinical and laboratory characteristics of patients cared for at a reference center in the south of Brazil. Jornal Brasileiro de Pneumologia 30:140-146, 2004.

8. Brito RC, Gounder C, Lima DB, Siqueira H, Cavalcanti HR, Pereira MM, Kritski AL. Resistência aos medicamentos anti-tuberculose de cepas de Mycobacterium tuberculosis isoladas de pacientes atendidos em hospital geral de referência para tratamento de AIDS no Rio de Janeiro. Jornal Brasileiro de Pneumologia 30:335-342, 2004.
9. Coelho AGV, Zamarioli LA, Reis CMPV, Figueiredo TAR. Avaliação dos testes empregados no diagnóstico laboratorial da tuberculose em pacientes HIV positivos. Revista do Instituto Adolfo Lutz 63:111-115, 2004.

10. Conde MB, Figueira CM, Moraes R, Fonseca LS, Deriemer K, Kritski AL. Predictive value of the acid fast smear for detection of Mycobacterium tuberculosis in respiratory specimens in a Reference Center of HIV/Aids in Rio de Janeiro, Brazil. Memórias do Instituto Oswaldo Cruz 94:787-790, 1999

11. Falkinham JO. Epidemiology of infection by nontuberculous mycobacteria. Clinical Microbiology Reviews 9:177-215, 1999.

12. Ferreira RMC, Saad MHF, Silva MG, Fonseca LS. Non-tuberculous Mycobacteria I: One Year Clinical Isolates Identification in Tertiary Hospital Aids Reference Center, Rio de Janeiro, Brazil, in Pre Highly Active Antiretroviral Therapy Era. Memórias do Instituto Oswaldo Cruz 97:725-729, 2002

13. Figueiredo RCPS. Estudo da utilização do método bacteriológico no diagnóstico da tuberculose pulmonar no município de Taubaté, S. Paulo. Dissertação de Mestrado. Faculdade de Saúde Pública, Universidade de São Paulo. São Paulo, SP, 1996.

14. Froes GC, Coutinho RL, Ávila MN, Cançado LR, Spindola de Miranda S. Perfil e seguimento dos pacientes portadores de Mycobacterium $s p$ do Hospital das Clínicas da Universidade Federal de Minas Gerais. Jornal de Pneumologia 29:365-370, 2003

15. Fundação Nacional de Saúde. Centro de Referência Prof. Hélio Fraga. Sociedade Brasileira de Pneumologia e Tisiologia. FUNASA/CRPHF/SBPT. Controle da Tuberculose: uma proposta de integração ensino-serviço. $5^{\text {a }}$ edição. Ministério da Saúde. Rio de Janeiro, 2002.

16. Fundação Nacional da Saúde. Programa Nacional de Combate à Tuberculose Manual de Bacteriologia da Tuberculose. $3^{\text {a }}$ edição. Ministério da Saúde. Rio de Janeiro, 2005.

17. Getahun H, Harrington M, O'Brien R, Nunn P. Diagnosis of smear-negative pulmonary tuberculosis in people with HIV infection or AIDS in resourceconstrained settings: informing urgent policy changes. Lancet 369: 2042-2049, 2007

18. Gomes CID, Rovaris DB, Severino JL, Gruner MF. Perfil de resistência de M. tuberculosis isolados de pacientes portadores do HIV/AIDS atendidos em um hospital de referência. Jornal de Pneumologia 26:25-29, 2000.

19. Harries AD, Mphasa NB, Mundy C, Banerjee A, Kwanjana JH, Salaniconi FML. Screening tuberculosis suspects using two sputum smears. International Journal of Tuberculosis and Lung Diseases 4:36-40, 2000.

20. Ipuge YAI, Rieder HL, Enarson DA. The yield of acid-fast bacilli from serial smears in routine microscopy laboratories in rural Tanzania. Transactions of the Royal Society of Tropical Medicine and Hygiene 90:258-261, 1996.

21. Jardim PCR, Zamarioli LA, Coelho AGV, Figueiredo TR, Rozman MA. Resistência do Mycobacterium tuberculosis às drogas no município de São Vicente. Revista do Instituto Adolfo Lutz 60:119-123, 2001.

22. Kritski A, Dalcomo M, Bianco R, Melo FF, Pinto WP, Sheehther M, Castelo A. Associação tuberculose e infecção pelo HIV no Brasil. Boletim de l'Oficina Sanitaria Panamericana 118:542-554, 1995

23. Liberato IRO, Albuquerque, LFM, Campelo ARL, Melo HRL. Characteristics of pulmonary tuberculosis in HIV seropositive and seronegative patients in a Northeastern region of Brazil. Revista da Sociedade Brasileira de Medicina Tropical 37:46-50, 2004

24. Marques M, Cunha EAT, Junqueira CT, Bernardes CA, Paniago AMM. Resistência do M. tuberculosis às drogas antituberculose entre pacientes HIV+ em Mato Grosso do Sul - Brasil. Jornal Brasileiro de Pneumologia 34 (S 1R): R30, 2008.

25. Melo FAF, Afiune JB, Ide Neto J, Almeida EA, Spada DTA, Antelmo ANL, Cruz ML. Aspectos epidemiológicos da tuberculose multirresistente em serviço de referência na cidade de São Paulo. Revista da Sociedade Brasileira de Medicina Tropical 36:27-34, 2003.

26. Ozkutuk A, Terek G, Coban H, Esen N. Is it valuable to examine more than one sputum smear per patient for the diagnosis of pulmonary tuberculosis? Japanese Journal of Infectious Diseases 60: 73-75, 2007.

27. Pedro HSP, Silva MABR, Rossit ARB, Pereira MIF, Goloni MRA, Machado RLD, Franco $\mathrm{C}$, Cordeschi T, Fenley JC. Isolamento de espécies do gênero Mycobacterium $\mathrm{sp}$ entre pacientes portadores de HIV/AIDS em São José do Rio Preto, SP. Brazilian Journal of Infectious Diseases 11 (suppl 2): \$46, 2007. 
28. Pinto WP, Hadad DJ, Palhares MCA, Ferrazoli L, Telles MAS, Ueki SYM, Santos MTF, Placco ALN, Sauaia N, Palaci M. Drug resistance of M. tuberculosis isolated from patients with HIV infection seen at an AIDS Reference Center in São Paulo, Brazil. Revista do Instituto de Medicina Tropical de São Paulo 38:15-21, 1996.

29. Rozman LM, Santo AH, Rozman, MA. Mycobacterium tuberculosis drug resistance in HIV patients in Baixada Santista, São Paulo, Brazil. Cadernos de Saúde Pública 23:1051-1059, 2007.

30. Rujula MJP, Galesi VMN. Análise da tendência da coinfecção tuberculose/hiv no Estado de São Paulo. Jornal Brasileiro de Pneumologia 34(supl 1R): R69-R70, 2008

31. Santi LQ, Valentini MB, Fonseca VF, Garcia GF, Eládio MA, Siqueira AEL. Análises das culturas para micobactérias de pacientes do Hospital Eduardo Menezes FHEMIG - BH. Brazilian Journal of Infectious Diseases 5 (S 2):S58, 2001.

32. Secretaria de Estado da Saúde. Boletim Epidemiológico Regional DIR XIX Baixada Santista. CRTDST/AIDS. nº 01, São Paulo, ano 2005. [acesso em 25 ago 2006]. Disponível em: http://www.crt.saude.sp.gov.br/arquivos/epidemio/ BOL_BAIXSANTISTA.pdf.

33. Secretaria de Estado da Saúde. Centro de Vigilância Epidemiológica. Divisão de Controle da Tuberculose. Micobacterioses: recomendações para o diagnóstico e tratamento. São Paulo, ano 2005. [acesso em 18 jul 2008]. Disponível em: http:// www.cve.saude.sp.gov.br/tuberculose/TUBERCULOSE/TUBERCULOSE_ORIENTA. htm

34. Secretaria de Estado da Saúde. Divisão de Tuberculose e Outras Pneumopatias. Tuberculose no Estado de São Paulo: Indicadores de Mortalidade e Indicadores de Desempenho. Boletim Epidemiológico Paulista. Suplemento 4. Ano 2006. Volume 3. [acesso em 18 julho 2008]. Disponível em http://www.cve.saude. sp.gov.br/agencia/bepa37_suple.htm
35. Secretaria de Políticas de Saúde. Manual técnico para o controle da tuberculose: cadernos de atenção básica. $6^{\text {a }}$ edição rev. e ampl. Departamento de Atenção Básica. Ministério da Saúde, Brasília, 2002.

36. Secretaria de Vigilância em Saúde. Departamento de Vigilância Epidemiológica. Doenças infecciosas e parasitárias: guia de bolso. $4^{\mathrm{a}}$ edição ampl. Ministério da Saúde, Brasília, 2004.

37. Seiscento M, Melo FAF, Ide Neto J, Noronha AML, Afiune JB, Inomata T, Cruz ML. Tuberculose multirresistente (TUBERCULOSEMR): aspectos clínico-laboratoriais, epidemiológicos e terapêuticos. Jornal de Pneumologia 23:237-244, 1997.

38. Telles MA, Ferrazoli 1, Waldman EA, Grampaglia CMS, Martins MC, Ueki SYM, Chimara E, Silva CA, Cruz V, Waldeman CCS, Heyn I, Uehara H, Riley LW. A population-based study of drug resistance and transmission of tuberculosis in an urban community. International Journal of Tuberculosis and Lung Diseases 9:1-7, 2005 .

39. Ueki SYM, Martins MC, Telles MAS, Virgilio MC, Giampaglia CMS, Chimara E, Ferrazoli L. Micobactérias não Tuberculosas: Diversidade das Espécies no Estado de São Paulo. Jornal Brasileiro de Patologia e Medicina Laboratorial 41:1-8, 2005.

40. Wolfart M, Barth AL, Willers D, Zavascki AP. Mycobacterium tuberculosis resistance in HIV-infected patients from a tertiary care teaching hospital in Porto Alegre, southern Brazil. Transactions of the Royal Society of Tropical Medicine and Hygiene 102: 421-425, 2008.

41. World Health Organization/HTM/Tuberculose. Tuberculose/HIV: a clinical manual. Geneva, 2004.

42. World Health Organization. HIV/AIDS. Reports on the Global AIDS Epidemic. Fact Sheet $\mathrm{n}^{0}$ 6, 2006. [acesso em 18 jul 2008]. Disponível em: http://www.who.int/ hiv/epiupdates/en/index.html. 